\title{
Variable Selection for High-Dimensional Genomic Data with Censored Outcomes Using Group Lasso Prior
}

\author{
Kyu Ha Lee ${ }^{\mathrm{a}, \mathrm{b}, *}$, Sounak Chakraborty ${ }^{\mathrm{c}}$, Jianguo Sun ${ }^{\mathrm{c}}$ \\ ${ }^{a}$ Epidemiology and Biostatistics Core, The Forsyth Institute, Cambridge, Massachusetts, U.S.A. \\ ${ }^{b}$ Department of Oral Health Policy and Epidemiology, Harvard School of Dental Medicine, Boston, \\ Massachusetts, U.S.A. \\ ${ }^{c}$ Department of Statistics, University of Missouri-Columbia, Columbia, Missouri, U.S.A.
}

\begin{abstract}
The variable selection problem is discussed in the context of high-dimensional failure time data arising from the accelerated failure time model. A data augmentation approach is employed in order to deal with censored survival times and to facilitate prior-posterior conjugacy. To identify a set of grouped relevant covariates, a shrinkage prior distribution is specified for the regression coefficients mimicking the effect of group lasso penalty. It is noted that unlike the corresponding frequentist method, a Bayesian penalized regression approach cannot shrink the estimates of coefficients to exact zeros in general. Toward resolving the issue, a two-stage thresholding method that exploits the scaled neighborhood criterion and the Bayesian information criterion is devised. Simulation studies are performed to assess the robustness and performance of the proposed method in terms of variable selection accuracy and predictive power. The method is successfully applied to a set of microarray data on the individuals diagnosed with diffuse large B-cell lymphoma. In addition, an $\mathrm{R}$ package called psbcGroup, which can be downloaded freely from CRAN, is developed for the implementation of the methods.
\end{abstract}

Keywords: Accelerated failure time model, Bayesian lasso, Gibbs sampler, Group lasso, Penalized regression

\section{Introduction}

Survival analysis with high-dimensional covariates has received substantial attention in the last few years. With the increasing ability for obtaining gene expression data for patients, genetic signatures have started to play a more important role than pathological outcomes in the study of the molecular portrait of a disease and the prediction of potential survival time (Sotiriou and Piccart, 2007). A comprehensive molecular and genetic profiling of the disease has a huge potential towards developing personalized medicine and targeted therapies.

*Corresponding author: 245 First St, Cambridge, MA 02142, U.S.A. Tel.: +1 617892 8549; fax: +16172624021 .

Email address: klee@forsyth.org (Kyu Ha Lee) 
In survival analysis, the most commonly used regression model is perhaps the proportional hazards $(\mathrm{PH})$ model (Cox, 1972). On the other hand, it is well-known that the PH model has some restrictions and may not fit the data well sometimes. Also in some contexts, the proportionality structure assumed by the model makes it hard to interpret regression coefficients (Wei, 1992; Hernán, 2010; Uno et al., 2015). To address these, many other models have been proposed and among them, one is the accelerated failure time (AFT) model (Prentice, 1978; Buckley and James, 1979 ; Koul et al., 1981). One important feature of the AFT model is that it provides a more direct physical interpretation on regression parameters (Collett, 2003) since it has a structure similar to the ordinary linear regression model and directly links the failure time of interest to covariates.

Variable selection in the high-dimensional setting has recently received a great deal of attention and many methods have been developed for it. Among them, one of the early and commonly used approach is the lasso penalized approach (Tibshirani, 1996) and by following it, other authors proposed a few other penalty functions including elastic-net (Zou and Hastie, 2005), grouped lasso (Yuan and Lin, 2006), and fused lasso (Tibshirani et al., 2005). Note that most of these and other existing methods were developed for either continuous response or binary response data, and also all of the penalization methods mentioned above are not based on any probabilistic framework. In addition, there are several penalization methods developed in the Bayesian paradigm (Park and Casella, 2008; Bornn et al., 2010; Kyung et al., 2010), which has the advantage that allows one to incorporate substantive prior information in the analysis. Also a Bayesian framework enables one to obtain the posterior predictive distribution and thus to readily quantify the uncertainty related to the prediction. Nevertheless, there only exists limited literature on Bayesian variable selection for high-dimensional data with the response variable of interest being a failure time or suffering right-censoring.

A few methods have been proposed for variable selection based on right-censored failure time data based on the Cox PH model (Tibshirani, 1997; Gui and Li, 2005; Bøvelstad et al., 2007; Tibshirani, 2009) and a nice comparative study for the methods can be found in Bøvelstad et al. (2007). Huang et al. (2006) and Engler and Li (2009) discussed some penalized approaches for the failure time data arising from the AFT model with a large number of predictors. Also in the Bayesian paradigm, Lee et al. (2011, 2015) developed variable selection methods for the PH model by using various shrinkage priors. Note that another traditional way to impose sparsity in the Bayesian paradigm is to specify spike and slab (a mixture of Normal and point mass distribution at zero) priors on regression coefficients. This is otherwise known as the stochastic search variable selection technique, studied extensively in George and McCulloch (1993, 1997) and applied in many applications (Brown et al. 1998; Sha et al., 2006; Hernández-Lobato et al., 2013; Narisetty and He, 2014). Newcombe et al. (2014) proposed a Bayesian variable selection method based on a reversible jump implementation of the Weibull model. Most recently, Zhang et al. (2016) developed a variable selection approach using lasso under AFT model, which studed the grouping structure among errors.

In the following, we will consider the analysis of right-censored failure time data 
arising from the AFT model and present a Bayesian method with the use of a shrinkage prior and the focus on the identification of a subset of important covariates related to the failure time of interest in the high dimensional situation $(n \ll p)$. In the method, to induce the sparsity of the model and group variable selection, we will utilize the scale mixture of normal and gamma distributions for regression coefficients that mimics the role of group lasso penalty and a data augmentation technique for the imputation of the censored failure times (Tanner and Wong, 1987; Komárek and Lesaffre, 2007). The prior specification along with the data augmentation approach can facilitate the prior-posterior conjugacy in the proposed Bayesian framework, and thus we can estimate the posterior distributions of parameters via typical Gibbs sampling without employing any complex Monte Carlo methods. Also the tuning parameter that controls the sparsity will be automatically adjusted and updated by specifying a hyperprior distribution. Note that although useful for shrinking regression parameters corresponding to irrelevant covariates toward zero, the Bayesian lasso does not have the ability to produce exact zero estimates like frequentist counterpart. In order to perform the variable selection, we will develop a two-stage thresholding method that accommodates both the posterior distribution of regression parameters and a goodness-of-fit.

The remainder of the paper is organized as follows. We will begin in Section 2 with introducing a motiving example, the diffuse large B-cell lymphoma (DLBCL) microarray study. In particular, some preliminary analysis results are provided and suggest that the PH model may not fit the data appropriately. Section 3 will present the proposed Bayesian inference procedure and Section 4 will discuss the variable selection problem and describe a thresholding method. In Section 5, simulation studies are conducted to assess the performance of the proposed methodology, especially the robustness, and the results suggest that the approach works well in practice. Section 6 applies the methodology to the DLBCL microarray study and some discussion and future research directions are given in Section 7 .

\section{Diffuse Large B-cell Lymphoma Microarray Study}

In this paper, we consider data collected from a DLBCL microarray study. Since molecular features of the tumors influences the mortality of patients with DLBCL after chemotherapy, Rosenwald et al. (2002) developed a molecular predictor of survival by using the gene-expression profiles of the lymphomas. In the study, a Lymphochip cDNA microarray with $p=7,399$ probes were used to monitor $n=240$ patients. The survival times for 102 subjects were right-censored. We applied the 10 nearestneighbors method for missing gene expressions in DLBCL data set.

Rosenwald et al. (2002) identified individual genes whose expression are correlated with patients' survival by using a Cox PH model. The PH model assumes constant hazard ratios over time. However, when the proportionality assumption is violated, the application of the Cox PH model has the potential to bias the results that may lead to the loss of power in estimation and inference of the prognostic factors on mortality (Therneau et al., 1990). Therefore, we check the PH assumption by adopting the Weibull heteroscedastic hazards regression model (Hsieh, 2001; Nikulin et al., 
2006). This approach permits the shape parameter of the Weibull baseline hazard function to depend upon covariate values. Let $T$ denote survival time and $\boldsymbol{x}$ a $p$ dimensional covariate vector. Specifically, we consider the following heteroscedastic Weibull model:

$$
\lambda\left(t_{i} ; \boldsymbol{x}_{i}, \boldsymbol{z}_{i}\right)=\eta \kappa_{0} e^{\boldsymbol{z}_{i}^{\top} \boldsymbol{\beta}^{*}} t_{i}^{\kappa_{0} e^{\boldsymbol{z}_{i}^{\top} \boldsymbol{\beta}^{*}}-1} e^{\boldsymbol{x}_{i}^{\top} \boldsymbol{\beta}^{0}}
$$

where $\eta$ and $\kappa_{i}=\kappa_{0} e^{\boldsymbol{z}_{i}^{\top} \boldsymbol{\beta}^{*}}$ are the scale and shape parameters of Weibull distribution and $\boldsymbol{z}_{i}$ is a vector of covariates for the $i^{\text {th }}$ patient associated with the shape parameter. $\boldsymbol{\beta}^{0} \in \mathbb{R}^{p}$ is a vector of regression parameters. Note that when $\boldsymbol{\beta}^{*}=0$, this model reduces to the conventional Weibull PH model. Therefore, the PH assumption can be tested under the nested Weibull PH model $\left(H_{0}: \beta_{k}^{*}=0\right.$ for the $k^{\text {th }}$ covariate $)$ within the model (1) $\left(H_{1}: \beta_{k}^{*} \neq 0\right)$ while setting $\boldsymbol{x}_{i}=\boldsymbol{z}_{i}$. We conducted a set of one-covariate analyses (i.e., including one covariate at a time in the model).
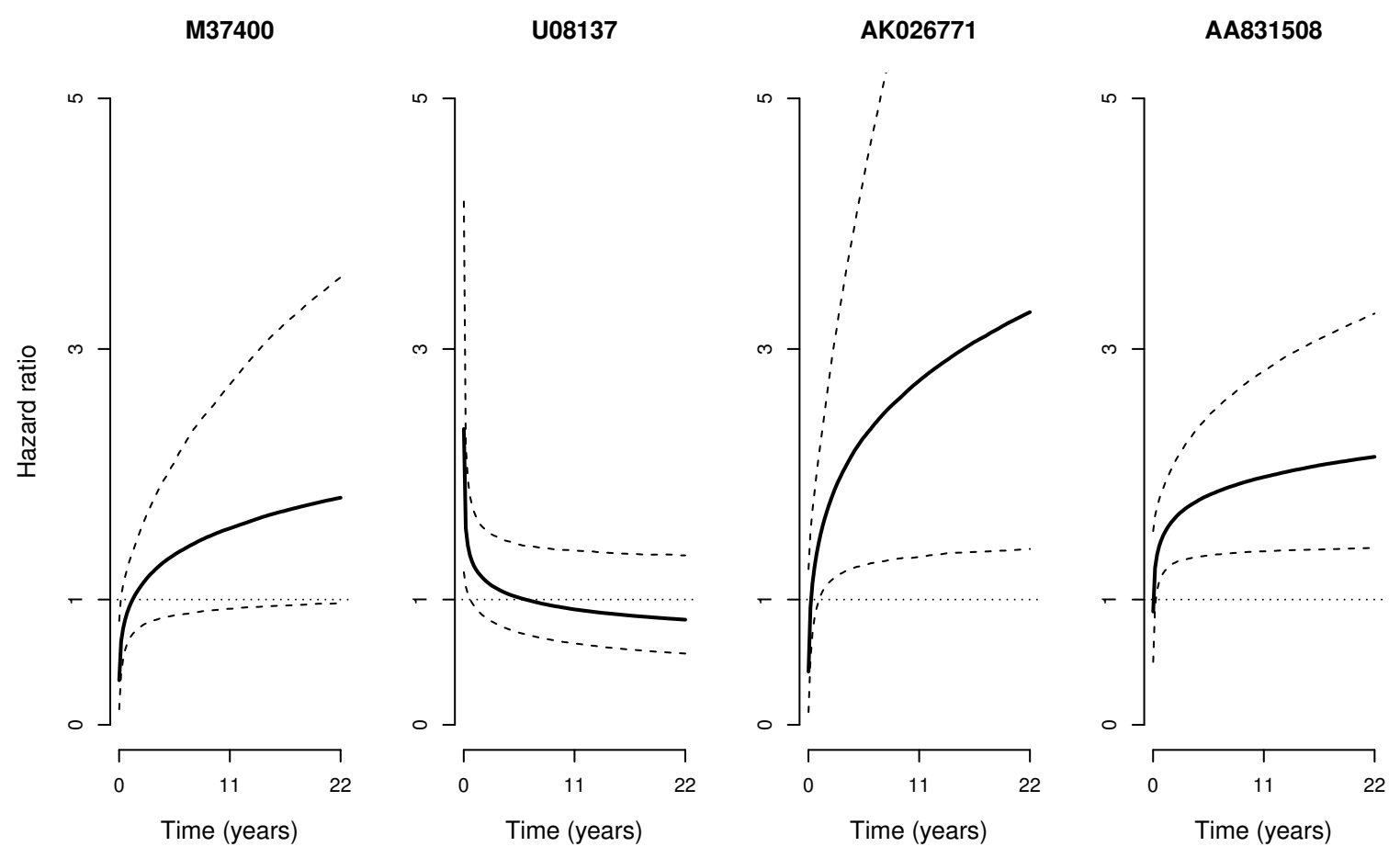

Figure 1: Pointwise posterior median and $95 \%$ credible intervals for the hazard ratio $(x=0$ vs 1) for the four genes associated with patients' mortality from the analysis of DLBCL data using the heteroscedastic Weibull model.

From the analyses, we found that the $\mathrm{PH}$ assumption is violated for the effect of 71 gene expressions on the patients survival under the heteroscedastic Weibull model. We present the estimates of $\beta^{*}$ and $\beta^{0}$ corresponding to four randomly selected genes out of the 71 genes in Table 1. We also provide the estimated hazard ratios over time for the four genomic variables ( $x=0$ vs 1 ) in Figure 1 . If the $\mathrm{PH}$ assumption is appropriate for a covariate, the hazard ratio must be constant over time. Therefore, Figure 1 gives immediate visual indication that a violation of the $\mathrm{PH}$ assumption is 
to be concerned for the analysis of DLBCL data. We note, however, that since the one-covariate analyses are only capable of marginally testing the $\mathrm{PH}$ assumption, the violation of the $\mathrm{PH}$ assumption found for the 73 genomic variables might be alleviated when being jointly considered with other variables. Unfortunately, to our knowledge, we are not aware of any conventional method to jointly test the PH assumption for survival data with high-dimensional covariates such as DLBCL data.

Table 1: Posterior medians (PM) and 95\% credible intervals (CI) for $\beta^{*}$ and $\beta^{0}$ for the four genes (NCBI, 2016) from the single covariate analyses using the heteroscedastic Weibull model.

\begin{tabular}{llcr}
\hline Genbank ID & \multirow{2}{*}{ Gene symbol } & $\beta^{*}$ & $\beta^{0}$ \\
& & PM $(95 \%$ CI $)$ & PM $(95 \%$ CI $)$ \\
\hline M37400 & GOT1 & $-0.16(-0.29,-0.01)$ & $0.32(0.10,0.53)$ \\
U08137 & FASLG & $0.16(0.04,0.28)$ & $-0.10(-0.32,0.11)$ \\
AK026771 & RALGAPB & $0.13(0.01,0.27)$ & $-0.15(-0.34,0.06)$ \\
AA831508 & & $0.19(0.05,0.34)$ & $-0.05(-0.26,0.16)$ \\
\hline
\end{tabular}

\section{A Bayesian AFT Model for High-Dimensional Survival Data}

In this section, we will present a Bayesian AFT model for the analysis of rightcensored failure time data with high-dimensional covariates. First we will describe the model and the prior distribution. The posterior distribution is then derived and followed by some discussion of its estimation.

\subsection{Notation, Model and Prior Distributions}

Consider a failure time study and let $T$ and $\boldsymbol{x}$ denote the failure time of interest and a $p$-dimensional covariate vector. The AFT model assumes that the covariates have some multiplicative effects on $T$ through the log-linear transformation

$$
W=\log (T)=\alpha+\boldsymbol{x}^{\top} \boldsymbol{\beta}+\varepsilon,
$$

where $\alpha$ is the intercept term, $\boldsymbol{\beta} \in \mathbb{R}^{p}$ is a vector of regression parameters, and the error term $\varepsilon$ is assumed to follow $\operatorname{Normal}\left(0, \sigma^{2}\right)$. Note that the regression parameters $\boldsymbol{\beta}$ play an important role in variable selection in penalized regression models.

Now we discuss the specification of the prior distribution. In a Bayesian framework, two major choices for the prior of $\boldsymbol{\beta}$ have been the Normal distribution with zero mean and the Laplace distribution which, respectively, correspond to ridge and lasso penalty in the frequentist penalized regression. In the proposed model, we will adopt a conditional Laplace prior that shrinks the regression coefficient corresponding to irrelevant variables towards zero.

Ordinary lasso prior (Park and Casella, 2008) can be expressed as a conditional Laplace prior of form:

$$
\pi\left(\boldsymbol{\beta} \mid \sigma^{2}\right) \propto \exp \left(-\frac{\lambda}{\sqrt{\sigma^{2}}} \sum_{j=1}^{p}\left|\beta_{j}\right|\right)
$$


The prior of $\boldsymbol{\beta}$ being conditioned on $\sigma^{2}$ is important not only because it makes updating model parameters exploit prior-posterior conjugacies in full conditionals but also because it guarantees a unimodal full posterior in linear regression analysis (Park and Casella, 2008). Following Andrews and Mallows (1974), the conditional Laplace distribution (3) can be expressed as a scaled mixture of normal and gamma distributions:

$$
\boldsymbol{\beta} \mid \sigma^{2}, \tau_{1}^{2}, \ldots, \tau_{p}^{2} \sim \operatorname{MVN}\left(\mathbf{0}_{p}, \sigma^{2} \boldsymbol{A}_{\boldsymbol{\tau}}\right), \quad \pi\left(\tau_{1}^{2}, \ldots, \tau_{p}^{2} \mid \lambda^{2}\right) \propto \prod_{j=1}^{p} \frac{\lambda^{2}}{2} e^{-\lambda^{2} \tau_{j}^{2} / 2} d \tau_{j}^{2},
$$

where $\boldsymbol{A}_{\boldsymbol{\tau}}=\operatorname{diag}\left(\tau_{1}^{2}, \ldots, \tau_{p}^{2}\right)$ and $\mathbf{0}_{p}$ denotes the vector with $p$ zeros. As we can see in (4), the parameter $\sigma^{2}$ is a component of the overall variability in $\boldsymbol{\beta}$ while the variation in each individual $\beta_{j}$ is quantified by latent variables $\tau_{j}^{2}$ whose prior distribution is dictated by the turning parameter $\lambda^{2}$. As the value of $\lambda^{2}$ increases, the prior variance of $\tau_{j}^{2}$ gets smaller, and subsequently the prior of $\boldsymbol{\beta}$ is more concentrated around $\mathbf{0}_{p}$ implying a sparser model.

However, the ordinary lasso prior lacks the ability to accommodate certain known grouping information of covariates into the prior. In order to incorporate a physical dependence/grouping structure of covariates into a Bayesian hierarchical model, Kyung et al. (2010) devised a group lasso prior that corresponds to the frequentist group lasso regularization (Yuan and Lin, 2006). In our proposed Bayesian framework, we specify the group lasso prior for $\boldsymbol{\beta}$ given by the following conditional Laplace distribution:

$$
\pi\left(\boldsymbol{\beta} \mid \sigma^{2}\right) \propto \exp \left(-\frac{\lambda}{\sqrt{\sigma^{2}}} \sum_{k=1}^{K}\left\|\boldsymbol{\beta}_{k}\right\|_{G_{k}}\right)
$$

where $K$ is the number of groups, $G_{k}$ 's are given positive definite matrices, and $\|\boldsymbol{\beta}\|_{G}=\left(\boldsymbol{\beta}^{\top} G \boldsymbol{\beta}\right)^{1 / 2} . \boldsymbol{\beta}_{k}$ is the $m_{k} \times 1$ subvector of $\boldsymbol{\beta}$ for group $k$, where $m_{k}$ is the size of the group. For $G_{k}=I_{m_{k}}$, where $I_{m_{k}}$ is the $m_{k} \times m_{k}$ identity matrix, we write $\left\|\boldsymbol{\beta}_{k}\right\|=\left\|\boldsymbol{\beta}_{k}\right\|_{I_{m_{k}}}$ for brevity. Covariates need to be classified a priori into $K$ groups, each of size $m_{k}$. The group lasso prior for $\boldsymbol{\beta}_{k}$ can also be expressed as a scaled mixture of Normal and Gamma distribution given by

$$
\boldsymbol{\beta}_{k} \mid \sigma^{2}, \tau_{k}^{2} \sim \operatorname{MVN}\left(\mathbf{0}_{m_{k}}, \sigma^{2} \tau_{k}^{2} I_{m_{k}}\right), \pi\left(\boldsymbol{\tau}^{2} \mid \lambda^{2}\right) \propto \prod_{k=1}^{K} \frac{\lambda^{2}}{2}\left(\tau_{k}^{2}\right)^{\left(m_{k}+1\right) / 2-1} e^{-\lambda^{2} \tau_{k}^{2} / 2}
$$

where $\mathbf{0}_{m_{k}}$ is the vector of $m_{k}$ zeros and $\boldsymbol{\tau}^{2}=\left(\tau_{1}^{2}, \ldots, \tau_{K}^{2}\right)$. The regression parameters in the group $k$ share the group-specific prior variance $\left(\sigma^{2} \tau_{k}^{2}\right)$, which gives rise to the effect of the frequentist group lasso penalty. For remaining unknown (hyper-) parameters the following conjugate priors are specified:

$$
\begin{aligned}
\alpha \mid \sigma^{2} & \sim \operatorname{Normal}\left(\alpha_{0}, h_{0} \sigma^{2}\right), \\
\sigma^{2} & \sim \operatorname{Inverse-Gamma}\left(\nu_{0} / 2, \nu_{0} \sigma_{0}^{2} / 2\right), \\
\lambda^{2} & \sim \operatorname{Gamma}(r, \delta),
\end{aligned}
$$

where $h_{0}, \nu_{0}, \sigma_{0}^{2}, r, \delta$ are the hyperparameters that require specification by the analyst. 


\subsection{The Joint Posterior Distribution}

When $t_{i}$ is subject to right-censoring, we can only observe $y_{i}=\min \left(t_{i}, c_{i}\right)$ and $\delta_{i}=I\left(t_{i} \leq c_{i}\right)$, where $c_{i}$ is the censoring time. In order to impute the censored outcomes, we adopt the augmentation approach (Tanner and Wong, 1987). Let $w_{i}=\log \left(t_{i}\right)$ be the augmented data such that

$$
\begin{array}{ll}
w_{i}=\log \left(y_{i}\right), & \text { if } \delta_{i}=1, \\
w_{i}>\log \left(y_{i}\right), & \text { if } \delta_{i}=0 .
\end{array}
$$

Let $\boldsymbol{D}$ denote the observed data from $n$ subjects: $\boldsymbol{D}=\{\boldsymbol{y}, \boldsymbol{\delta}, \boldsymbol{X}\}$, where $\boldsymbol{y}=\left(y_{1}, \ldots\right.$, $\left.y_{n}\right)^{\top}, \boldsymbol{\delta}=\left(\delta_{1}, \ldots, \delta_{n}\right)^{\top}$, and $\boldsymbol{X}$ is a $n \times p$ covariate matrix with $i$-th row vector being $\boldsymbol{x}_{i}$. The augmented data likelihood $\pi\left(w_{i} \mid \boldsymbol{D}, \alpha, \boldsymbol{\beta}, \sigma^{2}\right)$ is a Normal distribution:

$$
\operatorname{Normal}\left(\alpha+\boldsymbol{x}_{i}^{\top} \boldsymbol{\beta}, \sigma^{2}\right),
$$

with truncation given by (8). Combining (2), (6), and (7), the joint posterior distribution can be written as

$$
\begin{aligned}
& \pi\left(\boldsymbol{w}, \alpha, \boldsymbol{\beta}, \sigma^{2}, \boldsymbol{\tau}^{2}, \lambda^{2} \mid \boldsymbol{D}\right) \\
\propto & \prod_{i=1}^{n} \pi\left(w_{i} \mid \boldsymbol{D}, \alpha, \boldsymbol{\beta}, \sigma^{2}\right) \prod_{k=1}^{K} \pi\left(\boldsymbol{\beta}_{k} \mid \sigma^{2}, \tau_{k}^{2}\right) \pi\left(\tau_{k}^{2} \mid \lambda^{2}\right) \pi\left(\alpha \mid \sigma^{2}\right) \pi\left(\sigma^{2}\right) \pi\left(\lambda^{2}\right),
\end{aligned}
$$

where $\boldsymbol{w}=\left(w_{1}, \ldots, w_{n}\right)$.

Based on the data augmentation approach and our choice of prior specification, the conditional posterior distributions of all model parameters have standard forms. Therefore, the posterior distributions can be estimated by the efficient Gibbs sampling method. We provide the algorithm in the psbcGroup package for $\mathrm{R}$; the software is available from the Comprehensive $\mathrm{R}$ Archive Network (http://cran.rproject.org/web/packages/psbcGroup/). A detailed step-by-step Gibbs sampling algorithm and the full conditionals for each parameter are given in Appendix A.

\section{Thresholding method for Variable Selection}

As mentioned above, in contrast to the frequentist counterparts, the Bayesian lasso cannot produce the exact zero estimates for the regression parameters and therefore one needs a thresholding method to perform variable selection. The Bayes factors (BF, Kass and Raftery, 1995) have been widely used to quantify the evidence for model comparison. Schwarz et al. (1978) derived the Bayesian information criterion (BIC) as a large sample approximation to $2 \cdot \log (\mathrm{BF})$ and Volinsky and Raftery (2000) further showed that when there is censoring, replacing $n$ by the number of uncensored cases, $d$, in the penalty term of BIC formula in the expression (11) improves the approximation. This adjusted formula of the BIC for survival models has been used in many applications (among others, see chapter 6.5 of Ibrahim et al., 2005; Lee et al., 2011, 2015). 
However, the BIC thresholding solely relies on the point estimates of parameters and tends to disregard the associated uncertainties as shown in (11). Lee et al. (2015) also suggested using scaled neighborhood criterion (SNC) (Li and Lin, 2010) to perform variable selection: a covariate is selected if the posterior probability $P\left\{\left|\beta_{j}\right|>\right.$ $\left.\sqrt{\operatorname{var}\left(\beta_{j} \mid \boldsymbol{D}\right)} \mid \boldsymbol{D}\right\}$ exceeds a certain threshold, $\psi$. Although the SNC thresholding exploits the entire posterior distributions of $\boldsymbol{\beta}$ in variable selection, it is not straightforward how to determine the value of $\psi$.

Therefore, we propose a two-stage thresholding method called $S N C$-BIC, that utilizes the posterior distribution of regression parameters based on SNC method to select a group of candidate models for different values of $\psi$ and identify the best model based on the lowest BIC value:

1. Implement the algorithm provided in Appendix A to obtain $\tilde{\boldsymbol{\beta}}$, the posterior estimates of $\boldsymbol{\beta}$, for the full model.

2. Find $M$ candidate models based on SNC method over the grid of $\psi,\left\{\psi: \psi=\frac{m}{M}\right.$, $m=1, \ldots, M\}$. Note that depending on the grid of $\psi$, the number of unique candidate models may be less than $M$ because a range of $\psi$ values might lead to the same model.

3. It is noted that the posterior distribution of $\boldsymbol{\beta}$ will shift toward zero for a large value of $\lambda$. Therefore, if the estimation of $\boldsymbol{\beta}$ is the main goal, we use the scaled estimate given by $\hat{\gamma} \tilde{\boldsymbol{\beta}}$, where $\hat{\gamma}$ is obtained by maximizing the likelihood under AFT model for the single covariate $\boldsymbol{x}^{\top} \tilde{\boldsymbol{\beta}}$ (Tibshirani, 2009). Let $l_{m}\left(\hat{\gamma}_{m}\right)$ and $l_{f}\left(\hat{\gamma}_{f}\right)$ denote the maximized log-likelihood under the single-covariate model with the covariate $\boldsymbol{x}_{i, m}^{\top} \tilde{\boldsymbol{\beta}}_{m}$ obtained from the model $m$ and the single-covariate model with the covariate $\boldsymbol{x}^{\top} \tilde{\boldsymbol{\beta}}$ obtained from the full model, respectively, where $\boldsymbol{\beta}_{m}$ and $\boldsymbol{x}_{i, m}$ are the subvectors of $\boldsymbol{\beta}$ and $\boldsymbol{x}_{i}$ corresponding to the included covariates in model $m$. Then we calculate $\mathrm{BIC}_{m}$ :

$$
\mathrm{BIC}_{m}=-2\left\{l_{m}\left(\hat{\gamma}_{m}\right)-l_{f}\left(\hat{\gamma}_{f}\right)\right\}+\left(p_{m}-p\right) \log (d)
$$

where $p_{m}$ is the number of variables selected by SNC method with $\psi=m / M$.

4. Select the final model $m$, where $m=\operatorname{argmin}_{m} \mathrm{BIC}_{m}$.

\section{Simulation Studies}

To evaluate the performance of the methodology proposed in the previous sections, a series of simulation studies have been conducted with the focus on the investigation of the gain in variable selection/prediction performance achieved by incorporating the group information through the prior of $\boldsymbol{\beta}$ while keeping the AFT model framework and other prior specification the same. To this end, we compared the proposed AFT model that adopts the group lasso prior (AFT-GL) to the model that adopts the ordinary lasso prior (AFT-OL) in terms of variable selection capability and prediction power. In addition, we assessed the robustness of the models against a violation of 
the model assumption and investigate the performance of the models depending on censoring rates.

\subsection{Set-up and Data Generation}

Gaussian samples of size $n=100$ with $p=1,000$ predictors are simulated. To create a grouped variable situation, a covariate vector $\boldsymbol{x}_{i}$ is generated as follows (Zou and Hastie, 2005): $x_{i j}=Z_{k}+\varepsilon_{j}, Z_{k} \sim N(0,1), k=1,2,3, j=\{5 k-l: l=0, \ldots, 4\}$ and $x_{i j} \sim N(0,1), j=16, \ldots, p$, where $\varepsilon_{j}$ 's are generated from independently identically distributed $N(0,0.01)$. That is, three equally important groups of relevant covariates are generated while other irrelevant covariates are independent of one another.

Data sets are generated under twelve different scenarios depending on the sign/size of the effect of relevant covariates and the censoring rates as well as the error distributions. While the log-survival time $w_{i}$ is generated under the model (2) with $\sigma^{2}=1$ in Scenario 1-2, we also consider the three different non-Normal distributions for $\epsilon$ to investigate how much the performance of the proposed model deteriorates when the model assumption is violated. Specifically we consider $\epsilon_{i} \sim 0.5 \cdot \operatorname{Normal}(0$, $1)+0.5 \cdot \operatorname{Normal}(0,0.01)$ in Scenario 3-4; a standardized Gamma distribution, $\epsilon_{i}=2\left(a_{i}-\right.$ $0.5) / \sqrt{0.5}$ with $a_{i} \sim \operatorname{Gamma}(0.5,1)$, in Scenario $5-6 ; \epsilon_{i} \sim 0.5 \cdot \operatorname{Normal}(-1,0.01)+0.5$. Normal $(1,0.01)$ in Scenario 7-8. We give the variety to the level of effects of important covariates on the outcomes; while we set $\beta_{j}=3, j=1, \ldots, 15$ in Scenarios 1,3,5 and 7, we set $\boldsymbol{\beta}=(-2,-1,0.5,1,2,-2,-1,0.5,1,2,-2,-1,0.5,1,2)^{\top}$ in Scenarios $2,4,6$, and 8. The intercept $\alpha$ is set to 5 . For Scenario 1-8, the censoring times are simulated from a uniform distribution such that about $30 \%$ of subjects are right-censored. Scenario 9, 10, 11, and 12 are the same as Scenario 1, 2, 5, and 6, respectively, except the higher right-censoring rates (about 50\%). We randomly generate 100 data sets under each simulation scenario.

For each of the 100 datasets under each of the twelve scenarios we fit the AFTGL and AFT-OL models. We set the hyperparameters to the same values for both models. For the intercept $\alpha$, we set $\left(\alpha_{0}, h_{0}\right)=\left(0,10^{6}\right)$ leading to a non-informative prior. For variance parameter $\sigma^{2}$, we set $\left(\nu_{0}, \sigma_{0}^{2}\right)=(0.5,0.5)$ so that the induced prior had a median 2.8 and $95 \%$ central mass between 0.07 and $4.6 \cdot 10^{5}$. For the tuning parameter $\lambda^{2}$, we set $(r, \delta)=(0.5,0.01)$. This choice corresponds to a diffuse hyperprior distribution with a mean of 50 and a variance of 5,000. Given the prior specifications, two independent chains were run for a total of 30,000 scans each with the first half taken as burn-in. We assessed the convergence of the Markov chains by inspecting trace plots of posterior samples as well as by calculating the potential scale reduction factor (PSR) (Gelman et al., 2003), specifically requiring the PSR to be less than 1.05 for all model parameters.

\subsection{Simulation Results}

As outlined in Section 4, we used SNC-BIC method for variable selection. In simulation studies, we can obtain four quantities based on the variables selected: TP (the number of relevant variables correctly included in the model), TN (the number of irrelevant variables correctly excluded from the model), FP (the number of irrelevant variables mistakenly included in the model), and FN (the number of relevant variables 
Table 2: Variable selection performance of two shrinkage priors under the AFT model for twelve different simulation scenarios with $p=1,000$ predictors; as outlined in Section 4, we used SNC-BIC method for variable selection; the operating characteristics, the number of variables selected $\left(p_{\text {sel }}\right)$, and the posterior means $(\mathrm{PM})$ of $\lambda^{2}$ are averaged over 100 simulated data sets (standard deviations in parentheses).

\begin{tabular}{|c|c|c|c|c|c|c|c|}
\hline \multirow{2}{*}{ Scenario } & \multirow{2}{*}{ Method } & \multicolumn{4}{|c|}{ Operating characteristic, $\%$} & \multirow[b]{2}{*}{$p_{\mathrm{sel}}^{\dagger}(\mathrm{SD})$} & \multirow{2}{*}{$\begin{array}{c}\lambda^{2} \\
\mathrm{PM}(\mathrm{SD})\end{array}$} \\
\hline & & TPR (SD) & TNR (SD) & PPV (SD) & NPV (SD) & & \\
\hline \multirow[b]{2}{*}{1} & AFT-OL & $48.3(11.6)$ & $99.4(0.4)$ & $59.1(16.4)$ & $99.2(0.2)$ & $13.2(4.8)$ & $20.6(7.7)$ \\
\hline & AFT-GL & $100.0(0.0)$ & $100.0(0.0)$ & $98.9(2.7)$ & $100.0(0.0)$ & $15.2(0.4)$ & $18.0(5.4)$ \\
\hline \multirow{2}{*}{2} & AFT-OL & $41.3(7.9)$ & $99.6(0.3)$ & $63.8(16.7)$ & $99.1(0.1)$ & $10.4(3.4)$ & $36.6(8.9)$ \\
\hline & AFT-GL & $87.1(9.2)$ & $100.0(0.0)$ & $98.7(3.0)$ & $99.8(0.1)$ & $13.3(1.6)$ & $34.3(6.1)$ \\
\hline \multirow{2}{*}{3} & AFT-OL & $41.0(12.4)$ & $99.4(0.3)$ & $54.5(13.7)$ & $99.1(0.2)$ & $11.7(3.9)$ & $21.5(9.5)$ \\
\hline & AFT-GL & $100.0(0.0)$ & $100.0(0.1)$ & $98.5(3.3)$ & $100.0(0.0)$ & $15.3(0.6)$ & $17.8(4.9)$ \\
\hline \multirow{2}{*}{4} & AFT-OL & $41.3(7.6)$ & $99.6(0.3)$ & $64.1(16.2)$ & $99.1(0.1)$ & $10.3(3.3)$ & $37.0(9.0)$ \\
\hline & AFT-GL & $93.1(6.7)$ & $100.0(0.0)$ & $99.7(1.3)$ & $99.9(0.1)$ & $14.0(1.0)$ & $32.5(5.1)$ \\
\hline \multirow{2}{*}{5} & AFT-OL & $39.9(12.3)$ & $99.4(0.4)$ & $53.6(16.0)$ & $99.1(0.2)$ & $11.8(4.3)$ & $21.0(8.2)$ \\
\hline & AFT-GL & $100.0(0.0)$ & $100.0(0.1)$ & $98.8(3.5)$ & $100.0(0.0)$ & $15.2(0.6)$ & $18.9(4.8)$ \\
\hline \multirow{2}{*}{6} & AFT-OL & $39.1(8.8)$ & $99.6(0.3)$ & $62.5(18.6)$ & $99.1(0.1)$ & $10.3(3.8)$ & $37.5(10.4)$ \\
\hline & AFT-GL & $83.3(8.3)$ & $100.0(0.0)$ & $99.6(1.5)$ & $99.7(0.1)$ & $12.5(1.3)$ & $33.6(6.1)$ \\
\hline \multirow{2}{*}{7} & AFT-OL & $39.5(12.8)$ & $99.5(0.3)$ & $56.8(16.4)$ & $99.1(0.2)$ & $11.1(4.0)$ & $20.0(9.6)$ \\
\hline & AFT-GL & $100.0(0.0)$ & $100.0(0.0)$ & $99.5(1.7)$ & $100.0(0.0)$ & $15.1(0.3)$ & $17.3(4.9)$ \\
\hline \multirow{2}{*}{8} & AFT-OL & $41.7(8.5)$ & $99.6(0.3)$ & $64.8(19.6)$ & $99.1(0.1)$ & $10.6(4.0)$ & $37.5(9.1)$ \\
\hline & AFT-GL & $91.6(7.3)$ & $100.0(0.0)$ & $99.3(2.1)$ & $99.9(0.1)$ & $13.9(1.2)$ & $33.5(5.6)$ \\
\hline \multirow[b]{2}{*}{9} & AFT-OL & $38.1(13.1)$ & $99.4(0.4)$ & $52.2(16.4)$ & $99.1(0.2)$ & $11.6(4.6)$ & $22.0(9.4)$ \\
\hline & AFT-GL & $99.9(0.7)$ & $100.0(0.1)$ & $98.1(3.8)$ & $100.0(0.0)$ & $15.3(0.6)$ & $18.9(5.9)$ \\
\hline \multirow{2}{*}{10} & AFT-OL & $38.3(9.4)$ & $99.5(0.3)$ & $57.4(19.8)$ & $99.1(0.1)$ & $10.9(3.7)$ & $37.8(10.6)$ \\
\hline & AFT-GL & $84.2(9.1)$ & $100.0(0.0)$ & $98.8(3.1)$ & $99.8(0.1)$ & $12.8(1.5)$ & $34.0(6.2)$ \\
\hline \multirow{2}{*}{11} & AFT-OL & $36.4(12.8)$ & $99.4(0.3)$ & $48.1(15.4)$ & $99.0(0.2)$ & $11.9(4.0)$ & $19.5(7.8)$ \\
\hline & AFT-GL & $99.5(2.0)$ & $100.0(0.1)$ & $97.3(4.8)$ & $100.0(0.0)$ & $15.4(0.9)$ & $18.9(6.0)$ \\
\hline \multirow{2}{*}{12} & AFT-OL & $36.8(9.3)$ & $99.5(0.4)$ & $55.1(16.5)$ & $99.0(0.1)$ & $10.8(4.2)$ & $36.1(9.6)$ \\
\hline & AFT-GL & $80.9(9.6)$ & $100.0(0.0)$ & $99.1(2.9)$ & $99.7(0.1)$ & $12.3(1.6)$ & $33.1(6.5)$ \\
\hline
\end{tabular}

$\dagger$ The number of variables that are truly associated with the outcome is $q=15$.

mistakenly excluded from the model). Then we assess the capability of a method to correctly select (or drop) relevant (or irrelevant) variables by calculating the true positive rate (TPR), the true negative rate (TNR), the positive predictive value (PPV), and the negative predictive value $(\mathrm{NPV}): \mathrm{TPR}=\mathrm{TP} / q, \mathrm{TNR}=\mathrm{TN} /(p-q), \mathrm{PPV}=$ $\mathrm{TP} /(\mathrm{TP}+\mathrm{FP}), \mathrm{NPV}=\mathrm{TN} /(\mathrm{TN}+\mathrm{FN})$, where $q$ is the number of variables that are truly associated with outcomes. If only the truly relevant variables are selected, all the four quantities should be $100 \%$.

In Table 2, we present the mean operating characteristics and the average number of selected variables for AFT-GL and AFT-OL under the twelve different simulation scenarios with $p=1000$ variables. It is not a surprise that we see the both models generally perform better when effects are stronger (Scenarios 1, 3, 5, 7, 9, 11) and when the model is correctly specified (Scenarios 1-2 comparing to Scenarios 3-8). It appears that both AFT-OL and AFT-GL with SNC-BIC thresholding produce sparse models across all the scenarios (10.3 13.2 variables for AFT-OL and 13.3 15.4 
(a) Scenario 1

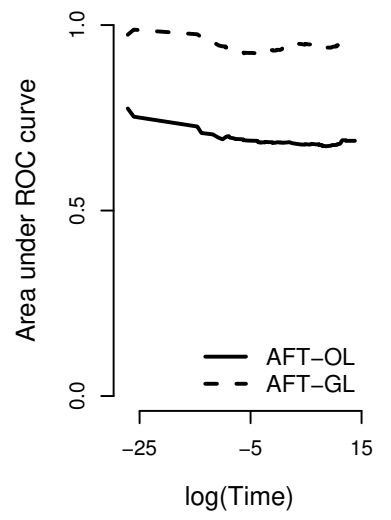

(e) Scenario 5

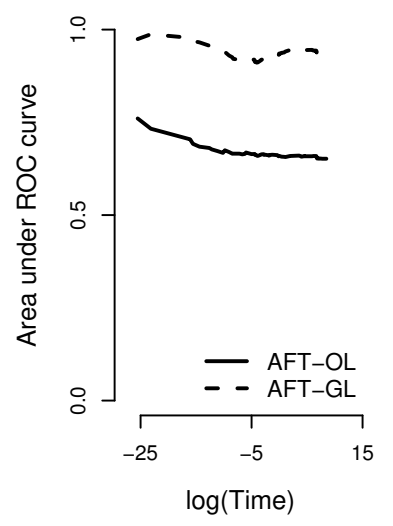

(i) Scenario 9

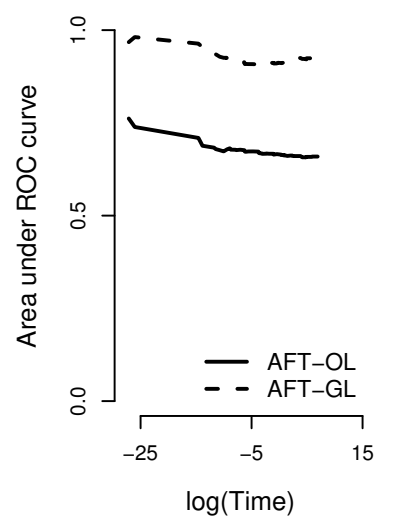

(b) Scenario 2

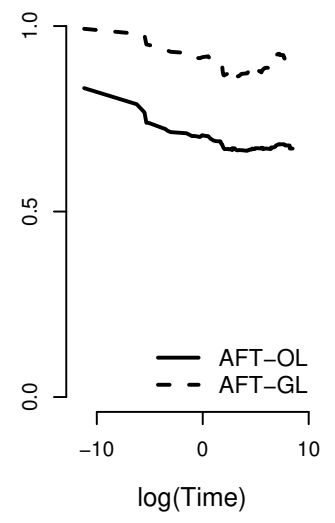

(f) Scenario 6

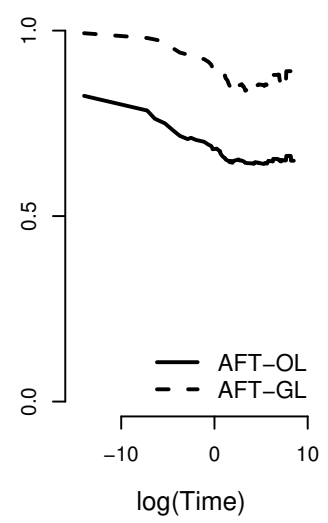

(j) Scenario 10

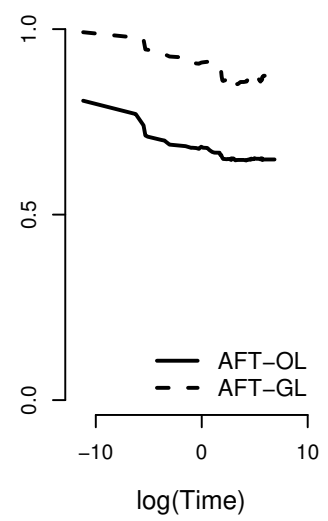

(c) Scenario 3

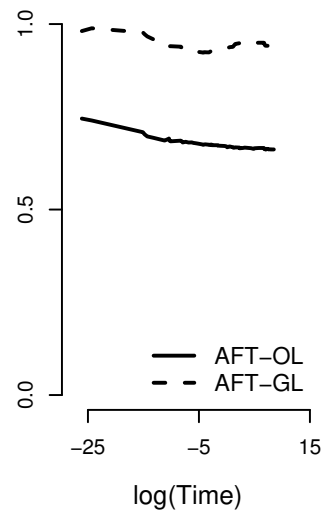

(g) Scenario 7

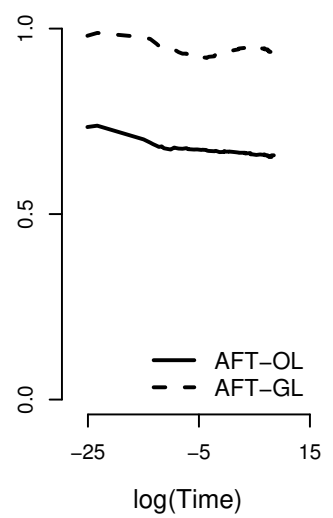

(k) Scenario 11

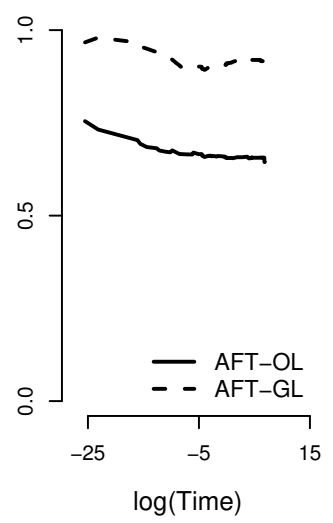

(d) Scenario 4

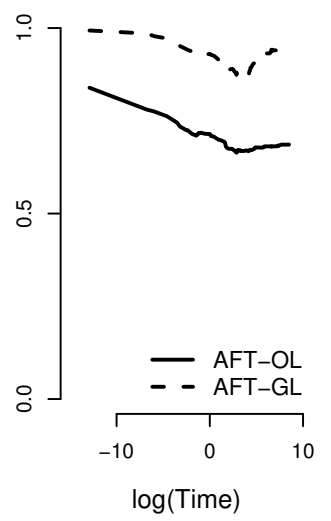

(h) Scenario 8

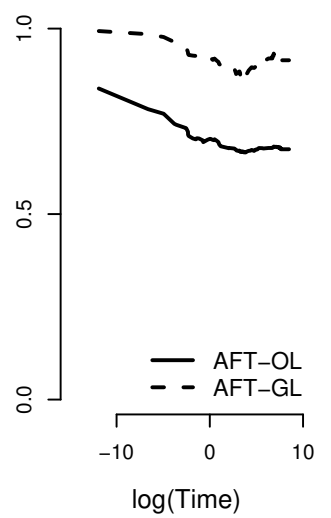

(I) Scenario 12

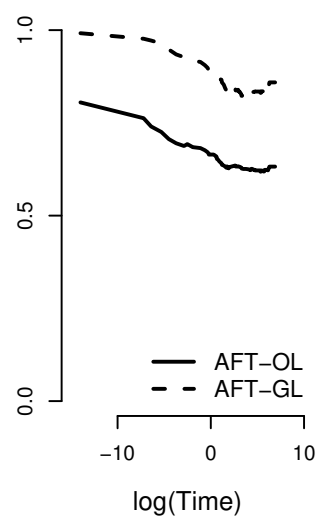

Figure 2: Prediction capability of AFT-OL and AFT-GL under twelve simulation scenarios: the mean area under time-dependent ROC curve plots from 100 simulated data sets.

variables for AFT-GL). We note, however, that AFT-OL yields much lower TPR and PPV as comparing to AFT-GL. For example, in Scenario 1, the TPR of $48.3 \%$ indicates that AFT-OL was able to detect only about 7 out of 15 truly relevant 
variables. Furthermore, only $59.1 \%$ of selected variables (PPV) are truly important variables. On the other hand, our proposed AFT-GL model yields much higher TPR and PPV (100\% and 98.9\%, respectively, in Scenario 1), indicating superior variable selection capability. Given our choice of the non-informative prior for $\lambda^{2}$, we found that AFT-OL generally selects less number of variables and fails to detect some true signals. As shown in Table 2, it can be in part due to the greater values for the estimated tuning parameter, $\lambda^{2}$, for AFT-OL which impose a stronger shrinkage than necessary.

We calculated the area under time-dependent ROC curve (AUC) (Heagerty et al., 2000) to evaluate the prediction accuracy for our proposed model. A test data set of size $n=100$ is generated under each of the twelve scenarios and the risk score $\boldsymbol{x}^{\top} \boldsymbol{\beta}$ is estimated for the test data set using the estimated $\boldsymbol{\beta}$ obtained from the training set. Then $\operatorname{ROC}(t \mid \boldsymbol{x})$ curve for time $t$ is drawn with coordinates $(\operatorname{TPR}(c, t \mid \boldsymbol{x})$, $1-\mathrm{TNR}(c, t \mid \boldsymbol{x}))$ with the cutoff $c$ varying. The time-dependent TPR and TNR for a given $c$ at time $t$ for a given risk score $\boldsymbol{x}^{\top} \boldsymbol{\beta}$ are defined as $P\left[\boldsymbol{x}^{\top} \boldsymbol{\beta}>c \mid \delta(t)=1\right]$ and $P\left[\boldsymbol{x}^{\top} \boldsymbol{\beta} \leq c \mid \delta(t)=0\right]$ respectively, where $\delta(t)$ is the event indicator at time $t$. Then AUC is the area under $\operatorname{ROC}(t \mid \boldsymbol{x})$ curve and the larger AUC at time $t$ indicates higher prediction power at time $t$. We provide the averaged AUC over 100 simulations in Figure 2 computed by AFT-OL for AFT-GL. Across all simulation scenarios, our proposed AFT-GL model shows better predictive performance over AFT-OL based on their consistently larger AUC values over time.

Our simulation studies exhibit that our proposed AFT-GL is relatively robust against the violation of the normal error assumption and higher censoring rates. As comparing to the ordinary lasso prior in AFT-OL model, the group lasso prior adopted in our proposed AFT-GL model provides more power to detect real effects by exploiting the grouping information.

\section{Application to DLBCL data}

Now we apply the methodology proposed in the previous sections to the DLBCL study described in Section 2 . For the intercept $\alpha$, we set $\left(\alpha_{0}, h_{0}\right)=\left(0,10^{6}\right)$ leading to a non-informative prior. We set $\left(\nu_{0}, \sigma_{0}^{2}\right)=(3,1)$ implying a weakly informative prior for $\sigma^{2}$ which is commensurate with the residual sum of squares obtained by the uncensored observations. We set $(r, \delta)=\left(0.5,10^{-3}\right)$ so that the prior for $\lambda^{2}$ is a non-informative distribution with a mean of 500 and a variance of $5 \cdot 10^{5}$. Given the prior specifications, two independent chains were run for a total of 50,000 scans each with the first half taken as burn-in.

As outlined in Section 3, AFT-GL requires grouping information. Following Ma et al. (2007), we calculated 'gap' statistics (Hastie et al., 2001) to determine the number of clusters. Then we applied the K-means clustering (Hartigan and Wong, 1979) to obtain group membership of the genes. The gap statistics suggested $K=4$ clusters for DLBCL data. Since K-means clustering may yield different configurations with different random seeds or starts, we conducted a sensitivity analysis. Indeed, given the number of clusters $(K=4)$, K-means clustering showed the variation in the resulting clustering configuration depending on the random seeds. We have summarized 


\section{Distribution of clustering configuration}

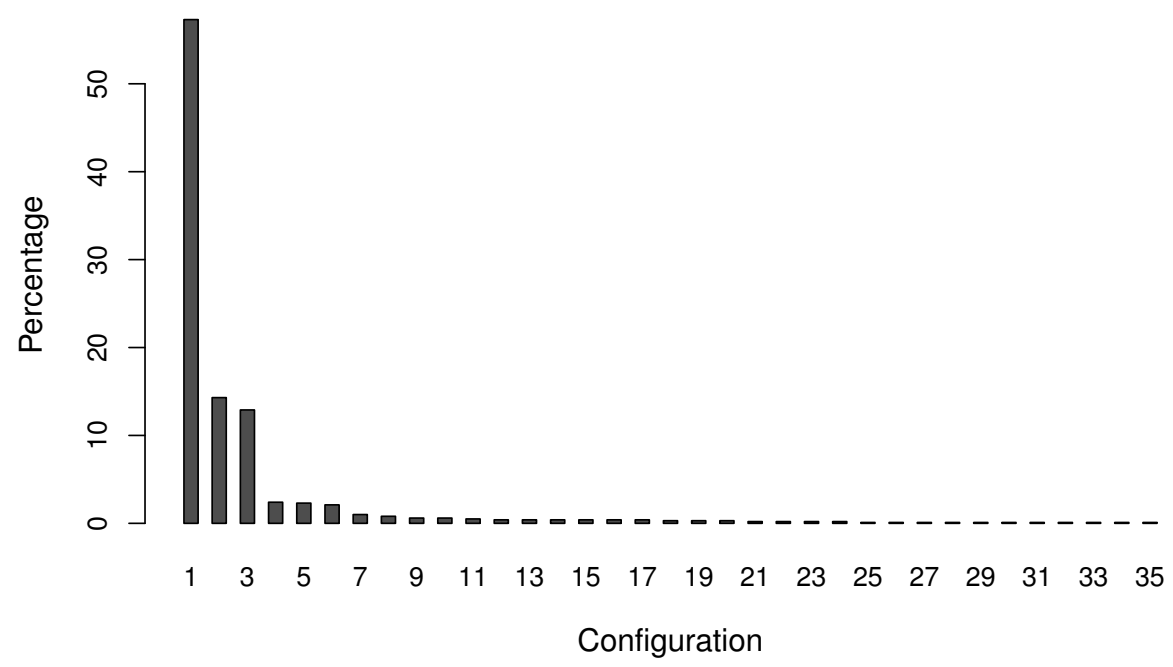

Figure 3: DLBCL data: distribution of clustering configuration using K-means clustering

the results in Figure 3. Among 1000 simulations, the configuration 1, which we have used for our analyses, was obtained about $58 \%$ of times.

To investigate whether any biological interpretation of the extracted 4 clusters, we categorize the 7399 genes based on the four signature groups defined by Rosenwald et al. (2002): Germinal-center B-cell (G), Lymph-node (L), Proliferation (P), and MHC class II (M). We provided the clustering information based on the signature groups in Table 3. Interestingly, we can see that Cluster 3 includes 97\% (347/357) of genes that belong to L signature group while Cluster 4 includes 87\% (1158/1333) of genes that belong to the $\mathrm{P}$ signature group. In addition, the majority (56\%) of genes in G signature group are classified into Cluster 2.

Table 3: Clustered gene expression information based on the four signature groups defined by Rosenwald et al. (2002): Germinal-center B-cell (G), Lymph-node (L), Proliferation (P), and MHC class II (M). We also provide the clustering information for Gene 1-6 identified by AFT-GL.

\begin{tabular}{ccccccc}
\hline Cluster & G & L & M & P & Undefined & Total \\
\hline 1 & 19 & 8 & 10 & 113 & $\begin{array}{c}2503 \\
(\text { Gene 3-6) }\end{array}$ & 2653 \\
2 & 84 & 1 & 0 & 57 & 1318 & 1460 \\
3 & 0 & 347 & 23 & 5 & 634 & 1009 \\
4 & 48 & 1 & 4 & $\begin{array}{c}1158 \\
(\text { Gene } 1)\end{array}$ & $\begin{array}{c}1066 \\
(\text { Gene 2) }\end{array}$ & 2277 \\
\hline Total & 151 & 357 & 37 & 1333 & 5521 & 7399 \\
\hline
\end{tabular}


Our proposed AFT-GL identified the six genes: AA521497 (Gene 1), Y16961 (Gene 2), M20681 (Gene 3), AF041261 (Gene 4), AA829241 (Gene 5), and AA808306 (Gene 6). Among the six identified genes, two of them belong to Cluster 4 and the other four genes belong to Cluster 1 as shown in Table 3. In addition, AFT-GL tends to select the genes that have high correlations: the pairwise sample corrections between (Gene 1, Gene 4, Gene 5) are 0.29, 0.22, 0.14 which are, respectively, 92nd, 83rd, and 70th percentiles of the sample corrections of all gene expressions in DLBCL data. We also note that none of the six genes correspond to the three genes, U84138, AA252328, LC31442, selected by the Bayesian Cox PH model with the group lasso prior (Lee et al., 2015), which reflects our initial investigation presented in Section 2. Finally, the posterior distribution of $\lambda$ has a median of 173.93 with $95 \%$ of central mass between 150.29 and 198.90 .

\section{DLBCL data}

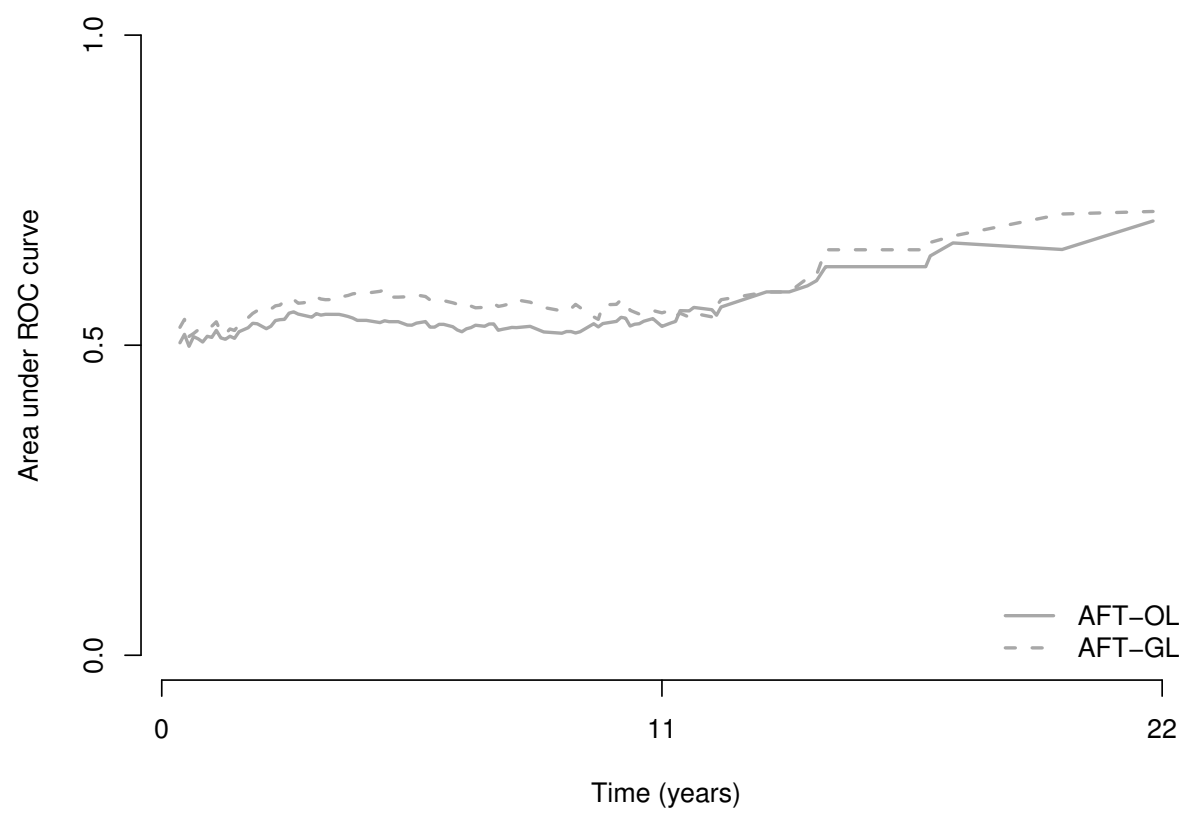

Figure 4: Predictability of AFT-OL and AFT-GL in DLBCL microarray data set: the mean area under time-dependent ROC curve from 10 random splits.

We also perform the analysis based on the AFT-OL to investigate the extent to which the Bayesian penalized AFT model could improve when exploiting the grouping information of covariates for the analysis of DLBCL data. We computed AUC for the two models to evaluate the prediction performance in analyses of DLBCL data. The data set is randomly split into a training and a test set (of size $\left.n_{\text {training }}=n_{\text {test }}=120\right) 10$ different times. Figure 4 provides the average AUC curves for the two fitting methods for DLBCL data. The AUC curve for our proposed AFT-GL is generally higher than that for AFT-OL, indicating the greater prediction power for our proposed AFT-GL. 


\section{Discussion and Future Researches}

In the preceding sections, a Bayesian AFT model has been proposed for the analysis of failure time data with high-dimensional covariates. As discussed above, the PH model can often fail in fitting real data, and the presented AFT-GL model provides a useful alternative and can exploit the information of the genes grouped by a clustering method or certain biological process through a group lasso shrinkage prior. In addition, an efficient computational scheme was developed and implemented in the psbcGroup R package.

Since the Bayesian penalized regression models do not perform the variable selection of itself, we proposed a two-stage thresholding method that takes into account both posterior variance of regression parameters and the model fit. In order to investigate whether our proposed SNC-BIC method shows an overall improvement, we perform the variable selection for the simulated data based on an empirical rule: selecting the variables that correspond to $\beta_{j}$ 's whose $95 \%$ credible intervals do not contain 0. The results are presented in Table B.4 in Appendix B. We can see that the empirical method tends to draw too conventional conclusions (i.e., too sparse) comparing results shown in Table 2 indicating the superior variable selection capability for the proposed SNC-BIC approach. In addition, in the simulations presented in Section 5, AFT-GL utilizes the true grouping information of the covariates, which is rarely available in the real situation. To investigate the uncertainty associated with the grouping structure, we implemented the AFT-GL model with grouping information estimated using gap statistics and K-means as done in Section6. The results are presented in Table B.5 in Appendix B. Although AFT-GL with the estimated grouping information doe not perform as well as that with the true grouping information, we can see that it still outperforms AFT-OL.

As seen in Section 5, the proposed AFT-GL consistently outperformed the AFTOL in the various situations including the cases when relatively higher percentage $(50 \%)$ of outcomes are right-censored. However, we note that the proposed computational scheme based on the data augmentation MCMC strategy had the potential to bias the results when a large amount of survival times are right-censored (i.e., there exists a strong determinant of survival times that is not included in the observed data). In the application to DLBCL microarray data where about $43 \%$ of outcomes are right-censored, our proposed AFT-GL model also shows a superior prediction power than AFT-OL model. While the proposed model demonstrated the robust performance in variable selection and prediction against the violation of normal error assumption in our numerical studies, the assumption might still be too simplistic for other applications where the underling survival distribution has more complex error structure. For example, in the simulation studies, when the covariate effects were relatively weak, the AFT-GL model yielded about 4\% lower TPR, indicating 0.6 less relevant covariates are included among selected, under the standardized gamma error distribution (Scenario 6) comparing to the case with normal error distribution (Scenario 2).

In order to provide more details on computation time and how realistically the proposed approach could scale up with $p$ and $n$, we also ran some simulation studies to 
check how the computation time grows as the number of covariates $p$ and the number of subjects $n$ increase, using a $2.5 \mathrm{GHz}$ Intel Core i7 MacBook Pro. We monitored the time to generate 10,000 MCMC scans for AFT-GL using the psbcGroup R package we built. The computations took about $0.2,3$, and 53 minutes for data from $n=100$ subjects with $p=100,1,000$ and 5,000, respectively and it took about 1, 5, and 74 minutes for data from $n=200$ subjects with $p=100,1,000$ and 5,000, respectively, indicating that our proposed algorithm is computationally efficient for data with highdimensional covariates. However, we note that a longer chain may be needed for data with many observations and high-dimensional covariates to make sure the posterior distribution samples achieve the convergence.

Finally note that in the application of AFT-GL model, it is required to obtain the grouping information before fitting the model. When considering genetic pathways to determine the grouping information of genes, it is typically the case that a set of genes belong to multiple pathways. Unfortunately, the group lasso prior considered in this paper cannot handle the problem because it can only deal with the groups that form a partition of the sets of variables. In the frequentist paradigm, Obozinski et al. (2011) proposed the overlap group lasso penalty that can identify sparse linear predictors whose supports are union of predefined overlapping groups of variables. Adapting the overlap group lasso penalty into Bayesian framework is a promising area for future development.

\section{Acknowledgements}

We would like to thank the University of Missouri Bioinformatics Consortium for letting us use their Dell EM64T Lewis cluster system. This work was supported partly by the National Science Foundation under the award number DMS-1106717.

\section{Appendix A. Computational Scheme}

In this section, we will describe an MCMC algorithm to fit our proposed Bayesian AFT model that adopts the group lasso prior outlined in Section 3. A detailed stepby-step Gibbs sampling algorithm and the full conditionals for each parameter are given as follows.

$\underline{\text { Step } 0}$ Start with initial values, $\boldsymbol{w}^{(0)}, \alpha^{(0)}, \boldsymbol{\beta}^{(0)}, \boldsymbol{\tau}^{2(0)}, \sigma^{2(0)}$, and $\lambda^{2(0)}$. The index $g$ denotes the $g^{\text {th }}$ iteration, and set $g=1$.

\section{The Imputation Step (Step 1)}

Step 1 It can be shown that the full conditional of $w_{i}$ with $\delta_{i}=0$ (right-censored observation) is a truncated Normal distribution given by (9). We use the algorithm proposed by Robert (1995) which avoids the long rejection time and thus enables efficient sampling from a truncated normal distribution.

$\underline{\text { The Posterior Steps (Step } 2 \text { - Step 6) }}$ 
$\underline{\text { Step } 2}$ The full conditional of $\alpha$ is a Normal distribution given by

$$
\alpha \mid \boldsymbol{D}, \boldsymbol{w}, \boldsymbol{\beta}, \sigma^{2}, \boldsymbol{\tau}^{2}, \lambda^{2} \sim \operatorname{Normal}\left(\frac{h_{0}(\boldsymbol{w}-\boldsymbol{X} \boldsymbol{\beta})^{\top} \mathbf{1}_{n}+\alpha_{0}}{n h_{0}+1}, \frac{\sigma^{2} h_{0}}{n h_{0}+1}\right) .
$$

Step 3 The full conditional of $\boldsymbol{\beta}_{k}, k=1, \ldots, K$, is a multivariate Normal distribution given by

$$
\begin{aligned}
& \boldsymbol{\beta}_{k} \mid \boldsymbol{D}, \boldsymbol{w}, \alpha, \boldsymbol{\beta}^{(-k)}, \sigma^{2}, \tau_{k}^{2}, \lambda^{2} \\
\sim & \operatorname{MVN}\left[\left(\boldsymbol{X}_{k}^{\top} \boldsymbol{X}_{k}+\frac{1}{\tau_{k}^{2}} I_{m_{k}}\right)^{-1} \boldsymbol{X}_{k}^{\top}\left(\boldsymbol{w}-\alpha \mathbf{1}_{n}-\frac{1}{2} \sum_{r \neq k} \boldsymbol{X}_{r} \boldsymbol{\beta}_{r}\right), \sigma^{2}\left(\boldsymbol{X}_{k}^{\top} \boldsymbol{X}_{k}+\frac{1}{\tau_{k}^{2}} I_{m_{k}}\right)^{-1}\right],
\end{aligned}
$$

where $\boldsymbol{\beta}^{(-k)}$ is the vector $\boldsymbol{\beta}$ with its subvector $\boldsymbol{\beta}_{k}$ removed and $\boldsymbol{X}_{k}$ is the submatrix of $X$ whose columns correspnd to $\boldsymbol{\beta}_{k}$. We note that the full conditional of $\boldsymbol{\beta}_{k}$ A.2 is the same as that given in Kyung et al. (2010) for linear regression setting.

Step 4 Since the full conditional of $\sigma^{2}$ is an inverse-gamma distribution, we draw a sample from

$$
\begin{aligned}
\sigma^{2} \mid \boldsymbol{D}, \boldsymbol{w}, \alpha, \boldsymbol{\beta}, \boldsymbol{\tau}^{2}, \lambda^{2} \sim & \text { Inverse-Gamma }\left(\frac{n+p+\nu_{0}+1}{2},\right. \\
& \left.\frac{1}{2}\left\{\nu_{0} \sigma_{0}^{2}+\frac{\left(\alpha-\alpha_{0}\right)^{2}}{h_{0}}+\left\|\boldsymbol{w}-\alpha \mathbf{1}_{n}-\boldsymbol{X} \boldsymbol{\beta}\right\|^{2}+\sum_{k=1}^{K} \frac{1}{\tau_{k}^{2}}\left\|\boldsymbol{\beta}_{k}\right\|^{2}\right\}\right) .
\end{aligned}
$$

Step 5 The full conditional of $1 / \tau_{k}^{2}, k=1, \ldots, K$, is an inverse-Gaussian distribution given by

$$
\frac{1}{\tau_{k}^{2}} \mid \boldsymbol{D}, \boldsymbol{w}, \alpha, \boldsymbol{\beta}, \sigma^{2}, \boldsymbol{\tau}^{2(-k)}, \lambda^{2} \sim \text { Inverse-Gaussian }\left(\sqrt{\frac{\lambda^{2} \sigma^{2}}{\left\|\boldsymbol{\beta}_{k}\right\|^{2}}}, \lambda^{2}\right) .
$$

Step 6 The full conditional for $\lambda^{2}$ is a gamma so we sample from

$$
\lambda^{2} \mid \boldsymbol{D}, \boldsymbol{w}, \alpha, \boldsymbol{\beta}, \sigma^{2}, \boldsymbol{\tau}^{2} \sim \operatorname{Gamma}\left(\frac{p+K}{2}+r, \frac{1}{2} \sum_{k=1}^{K} \tau_{k}^{2}+\delta\right) .
$$

Step 7 Let $G$ denote the number of posterior samples desired. If $g<G$, then $g=g+1$, and go to Step 2. Otherwise, go to the next step.

$\underline{\text { Step } 8}$ Calculate the posterior mean and median of $\boldsymbol{\beta}$ to obtain estimators. 


\section{Appendix B. Additional Simulation Results}

Table B.4: Variable selection performance of two shrinkage priors under the AFT model for twelve different simulation scenarios with $p=1,000$ predictors; as outlined in the Discussion, we used $95 \%$ credible intervals for variable selection; the operating characteristics and the number of variables selected $\left(p_{\text {sel }}\right)$ are averaged based on selected variables from 100 simulated data sets (standard deviations in parentheses).

\begin{tabular}{|c|c|c|c|c|c|c|}
\hline \multirow{2}{*}{ Scenario } & \multirow{2}{*}{ Method } & \multicolumn{4}{|c|}{ Operating characteristic, $\%$} & \multirow[b]{2}{*}{$p_{\mathrm{sel}}^{\dagger}(\mathrm{SD})$} \\
\hline & & TPR (SD) & TNR (SD) & $\mathrm{PPV}(\mathrm{SD})$ & NPV (SD) & \\
\hline \multirow{2}{*}{1} & AFT-OL & $0.1(0.9)$ & $100.0(0.0)$ & $\mathrm{NaN}^{*}$ & $98.5(0.0)$ & $<0.1(0.1)$ \\
\hline & AFT-GL & $54.1(13.0)$ & $100.0(0.0)$ & $100.0(0.0)$ & $99.3(0.2)$ & $8.1(2.0)$ \\
\hline \multirow{2}{*}{2} & AFT-OL & $0.2(1.1)$ & $100.0(0.0)$ & $\mathrm{NaN}$ & $98.5(0.0)$ & $<0.1(0.2)$ \\
\hline & AFT-GL & $41.8(6.3)$ & $100.0(0.0)$ & $100.0(0.0)$ & $99.1(0.1)$ & $6.3(0.9)$ \\
\hline \multirow{2}{*}{3} & AFT-OL & $0.0(0.0)$ & $100.0(0.0)$ & $\mathrm{NaN}$ & $98.5(0.0)$ & $0.0(0.0)$ \\
\hline & AFT-GL & $55.1(12.7)$ & $100.0(0.0)$ & $100.0(0.0)$ & $99.3(0.2)$ & $8.3(1.9)$ \\
\hline \multirow{2}{*}{4} & AFT-OL & $0.2(1.1)$ & $100.0(0.0)$ & $\mathrm{NaN}$ & $98.5(0.0)$ & $<0.1(0.2)$ \\
\hline & AFT-GL & $52.4(7.6)$ & $100.0(0.0)$ & $100.0(0.0)$ & $99.3(0.1)$ & $7.9(1.1)$ \\
\hline \multirow{2}{*}{5} & AFT-OL & $0.1(0.7)$ & $100.0(0.0)$ & $\mathrm{NaN}$ & $98.5(0.0)$ & $<0.1(0.1)$ \\
\hline & AFT-GL & $49.7(12.4)$ & $100.0(0.0)$ & $100.0(0.0)$ & $99.2(0.2)$ & $7.5(1.9)$ \\
\hline \multirow{2}{*}{6} & AFT-OL & $0.1(0.9)$ & $100.0(0.0)$ & $\mathrm{NaN}$ & $98.5(0.0)$ & $<0.1(0.1)$ \\
\hline & AFT-GL & $45.3(7.9)$ & $100.0(0.0)$ & $100.0(0.0)$ & $99.2(0.1)$ & $6.8(1.2)$ \\
\hline \multirow{2}{*}{7} & AFT-OL & $0.0(0.0)$ & $100.0(0.0)$ & $\mathrm{NaN}$ & $98.5(0.0)$ & $0.0(0.0)$ \\
\hline & AFT-GL & $54.5(13.2)$ & $100.0(0.0)$ & $100.0(0.0)$ & $99.3(0.2)$ & $8.2(2.0)$ \\
\hline \multirow{2}{*}{8} & AFT-OL & $0.4(1.6)$ & $100.0(0.0)$ & $\mathrm{NaN}$ & $98.5(0.0)$ & $0.1(0.2)$ \\
\hline & AFT-GL & $51.4(7.6)$ & $100.0(0.0)$ & $100.0(0.0)$ & $99.3(0.1)$ & $7.7(1.1)$ \\
\hline \multirow{2}{*}{9} & AFT-OL & $0.0(0.0)$ & $100.0(0.0)$ & $\mathrm{NaN}$ & $98.5(0.0)$ & $0.0(0.0)$ \\
\hline & AFT-GL & $18.5(11.0)$ & $100.0(0.0)$ & $100.0(0.0)$ & $98.8(0.2)$ & $2.8(1.6)$ \\
\hline \multirow{2}{*}{10} & AFT-OL & $0.1(0.9)$ & $100.0(0.0)$ & $\mathrm{NaN}$ & $98.5(0.0)$ & $<0.1(0.1)$ \\
\hline & AFT-GL & $33.0(9.0)$ & $100.0(0.0)$ & $100.0(0.0)$ & $99.0(0.1)$ & $5.0(1.4)$ \\
\hline \multirow{2}{*}{11} & AFT-OL & $0.0(0.0)$ & $100.0(0.0)$ & $\mathrm{NaN}$ & $98.5(0.0)$ & $0.0(0.0)$ \\
\hline & AFT-GL & $18.1(9.8)$ & $100.0(0.0)$ & $100.0(0.0)$ & $98.8(0.1)$ & $2.7(1.5)$ \\
\hline \multirow{2}{*}{12} & AFT-OL & $0.0(0.0)$ & $100.0(0.0)$ & $\mathrm{NaN}$ & $98.5(0.0)$ & $0.0(0.0)$ \\
\hline & AFT-GL & $30.7(9.8)$ & $100.0(0.0)$ & $100.0(0.0)$ & $99.0(0.1)$ & $4.6(1.5)$ \\
\hline
\end{tabular}

$\dagger$ The number of variables that are truly associated with the outcome is $q=15$.

* No variables are selected, i.e., PPV=0/0, for at least one of the splits. 
Table B.5: Variable selection performance of AFT-GL model for twelve different simulation scenarios with $p=1,000$ predictors when we estimate the grouping information based on the gap statistics and K-means clustering; as outlined in Section 4, we used SNC-BIC method for variable selection; the operating characteristics, the number of variables selected $\left(p_{\text {sel }}\right)$, and the posterior means (PM) of $\lambda^{2}$ are averaged over 100 simulated data sets (standard deviations in parentheses).

\begin{tabular}{ccccccc}
\hline \multirow{2}{*}{ Scenario } & \multicolumn{5}{c}{ Operating characteristic, \% } & \multicolumn{2}{c}{$\lambda^{2}$} \\
\cline { 2 - 5 } & TPR (SD) & TNR (SD) & PPV (SD) & NPV (SD) & $p_{\text {sel }}^{\dagger}(\mathrm{SD})$ & PM (SD) \\
\hline 1 & $59.0(14.7)$ & $99.7(0.3)$ & $78.4(16.6)$ & $99.4(0.2)$ & $11.9(4.2)$ & $11.0(7.7)$ \\
2 & $51.7(10.2)$ & $99.7(0.3)$ & $79.9(17.6)$ & $99.3(0.2)$ & $10.3(3.6)$ & $25.6(9.5)$ \\
3 & $59.7(13.6)$ & $99.7(0.3)$ & $76.8(14.5)$ & $99.4(0.2)$ & $12.1(3.8)$ & $12.4(6.8)$ \\
4 & $53.3(10.8)$ & $99.8(0.2)$ & $85.1(15.7)$ & $99.3(0.2)$ & $9.8(2.9)$ & $25.0(9.1)$ \\
5 & $60.3(12.3)$ & $99.6(0.3)$ & $74.7(16.1)$ & $99.4(0.2)$ & $12.7(4.0)$ & $12.7(7.5)$ \\
6 & $51.4(10.4)$ & $99.8(0.2)$ & $80.8(14.7)$ & $99.3(0.2)$ & $9.9(2.8)$ & $25.1(9.3)$ \\
7 & $59.1(14.8)$ & $99.7(0.3)$ & $77.4(15.8)$ & $99.4(0.2)$ & $12.0(4.0)$ & $12.7(8.6)$ \\
8 & $53.0(10.0)$ & $99.8(0.2)$ & $83.2(15.4)$ & $99.3(0.1)$ & $9.9(2.9)$ & $26.5(9.6)$ \\
9 & $51.1(14.4)$ & $99.7(0.3)$ & $75.6(17.4)$ & $99.3(0.2)$ & $10.8(4.2)$ & $11.6(5.9)$ \\
10 & $48.1(11.7)$ & $99.7(0.3)$ & $77.9(18.6)$ & $99.2(0.2)$ & $9.8(3.6)$ & $25.2(9.2)$ \\
11 & $51.5(15.1)$ & $99.6(0.3)$ & $71.2(19.5)$ & $99.3(0.2)$ & $11.6(4.3)$ & $12.2(7.5)$ \\
12 & $48.8(10.4)$ & $99.7(0.3)$ & $73.0(17.4)$ & $99.2(0.2)$ & $10.6(3.3)$ & $25.6(8.9)$ \\
\hline
\end{tabular}

$\dagger$ The number of variables that are truly associated with the outcome is $q=15$. 


\section{References}

Andrews, D., Mallows, C., 1974. Scale mixtures of normal distributions. Journal of the Royal Statistical Society. Series B (Methodological), 99-102.

Bornn, L., Gottardo, R., Doucet, A., 2010. Grouping priors and the bayesian elastic net. UBC Department of Statistics Technical Report \#254; Arxiv preprint arXiv:1001-4083.

Bøvelstad, H., Nygård, S., Størvold, H., Aldrin, M., et al., 2007. Predicting survival from microarray data: a comparative study. Bioinformatics 23 (16), 2080-2087.

Brown, P., Vannucci, M., Fearn, T., 1998. Multivariate Bayesian variable selection and prediction. Journal of the Royal Statistical Society: Series B 60 (3), 627-641.

Buckley, J., James, I., 1979. Linear regression with censored data. Biometrika 66 (3), 429-436.

Collett, D., 2003. Modelling survival data in medical research. CRC press.

Cox, D., 1972. Regression models and life-tables. Journal of the Royal Statistical Society. Series B (Methodological), 187-220.

Engler, D., Li, Y., 2009. Survival analysis with high-dimensional covariates: an application in microarray studies. Stat Appl Genet Mol Biol 8, 14.

Gelman, A., Carlin, J. B., Stern, H. S., Rubin, D. B., 2003. Bayesian data analysis. Chapman \& Hall/CRC.

George, E., McCulloch, R., 1993. Variable selection via Gibbs sampling. Journal of the American Statistical Association, 881-889.

George, E., McCulloch, R., 1997. Approaches for Bayesian variable selection. Statistica Sinica 7, 339-374.

Gui, J., Li, H., 2005. Penalized cox regression analysis in the high-dimensional and low-sample size settings, with applications to microarray gene expression data. Bioinformatics 21 (13), 3001-3008.

Hartigan, J., Wong, M., 1979. A k-means clustering algorithm. Applied statistics, 100.

Hastie, T., Tibshirani, R., Walther, G., 2001. Estimating the number of data clusters via the Gap statistic. J Roy Stat Soc B 63, 411-423.

Heagerty, P., Lumley, T., Pepe, M., 2000. Time-dependent ROC curves for censored survival data and a diagnostic marker. Biometrics 56 (2), 337-344.

Hernán, M. A., 2010. The hazards of hazard ratios. Epidemiology 21 (1), 13-15. 
Hernández-Lobato, D., Hernández-Lobato, J. M., Dupont, P., 2013. Generalized spike-and-slab priors for Bayesian group feature selection using expectation propagation. The Journal of Machine Learning Research 14 (1), 1891-1945.

Hsieh, F., 2001. On heteroscedastic hazards regression models: theory and application. Journal of the Royal Statistical Society: Series B 63 (1), 63-79.

Huang, J., Ma, S., Xie, H., 2006. Regularized estimation in the accelerated failure time model with high-dimensional covariates. Biometrics 62 (3), 813-820.

Ibrahim, J., Chen, M., Sinha, D., 2005. Bayesian survival analysis. Wiley Online Library.

Kass, R. E., Raftery, A. E., 1995. Bayes factors. Journal of the American Statistical Association 90 (430), 773-795.

Komárek, A., Lesaffre, E., 2007. Bayesian accelerated failure time model for correlated censored data with a normal mixture as an error distribution. Statistica Sinica 17, 549-569.

Koul, H., Susarla, V., Van Ryzin, J., 1981. Regression analysis with randomly rightcensored data. The Annals of Statistics, 1276-1288.

Kyung, M., Gill, J., Ghosh, M., Casella, G., 2010. Penalized regression, standard errors, and Bayesian lassos. Bayesian analysis 5 (2), 369-412.

Lee, K. H., Chakraborty, S., Sun, J., 2011. Bayesian variable selection in semiparametric proportional hazards model for high dimensional survival data. The International Journal of Biostatistics 7 (1), 1-32.

Lee, K. H., Chakraborty, S., Sun, J., 2015. Survival prediction and variable selection with simultaneous shrinkage and grouping priors. Statistical Analysis and Data Mining 8 (2), 114-127.

Li, Q., Lin, N., 2010. The Bayesian elastic net. Bayesian Analysis 5 (1), 151-170.

Ma, S., Song, X., Huang, J., 2007. Supervised group Lasso with applications to microarray data analysis. BMC bioinformatics 8 (1), 60.

Narisetty, N. N., He, X., 2014. Bayesian variable selection with shrinking and diffusing priors. The Annals of Statistics 42 (2), 789-817.

NCBI, 2016. National Center for Biotechnology Information: Gene. URL (accessed 29 January 2016): http://www.ncbi.nlm.nih.gov/gene/.

Newcombe, P., Ali, H. R., Blows, F., Provenzano, E., Pharoah, P., Caldas, C., Richardson, S., 2014. Weibull regression with Bayesian variable selection to identify prognostic tumour markers of breast cancer survival. Statistical Methods in Medical Research, DOI: 10.1177/0962280214548748 [Epub ahead of print]. 
Nikulin, M. S., Commenges, D., Huber, C., 2006. Probability, Statistics, and Modelling in Public Health. Springer.

Obozinski, G., Jacob, L., Vert, J.-P., 2011. Group lasso with overlaps: the latent group lasso approach. [Research Report] inria-00628498, page 60.

Park, T., Casella, G., 2008. The Bayesian lasso. Journal of the American Statistical Association 103 (482), 681-686.

Prentice, R. L., 1978. Linear rank tests with right censored data. Biometrika 65 (1), $167-179$.

Robert, C. P., 1995. Simulation of truncated normal variables. Statistics and computing 5 (2), 121-125.

Rosenwald, A., Wright, G., Chan, W., Connors, J., Campo, E., Fisher, R., et al., 2002. The use of molecular profiling to predict survival after chemotherapy for diffuse large-b-cell lymphoma. New England Journal of Medicine 346 (25), 19371947.

Schwarz, G., et al., 1978. Estimating the dimension of a model. The Annals of statistics $6(2), 461-464$.

Sha, N., Tadesse, M., Vannucci, M., 2006. Bayesian variable selection for the analysis of microarray data with censored outcomes. Bioinformatics 22 (18), 2262-2268.

Sotiriou, C., Piccart, M. J., 2007. Taking gene-expression profiling to the clinic: when will molecular signatures become relevant to patient care? Nature Reviews Cancer 7 (7), 545-553.

Tanner, M. A., Wong, W. H., 1987. The calculation of posterior distributions by data augmentation. Journal of the American statistical Association 82 (398), 528-540.

Therneau, T. M., Grambsch, P. M., Fleming, T. R., 1990. Martingale-based residuals for survival models. Biometrika 77 (1), 147-160.

Tibshirani, R., 1996. Regression shrinkage and selection via the lasso. Journal of the Royal Statistical Society. Series B (Methodological), 267-288.

Tibshirani, R., 1997. The lasso method for variable selection in the cox model. Statistics in medicine 16 (4), 385-395.

Tibshirani, R., 2009. Univariate shrinkage in the cox model for high dimensional data. Statistical Applications in Genetics and Molecular Biology 8 (1), 21.

Tibshirani, R., Saunders, M., Rosset, S., Zhu, J., Knight, K., 2005. Sparsity and smoothness via the fused lasso. Journal of the Royal Statistical Society: Series B (Statistical Methodology) 67 (1), 91-108. 
Uno, H., Wittes, J., Fu, H., Solomon, S. D., Claggett, B., Tian, L., Cai, T., Pfeffer, M. A., Evans, S. R., Wei, L.-J., 2015. Alternatives to hazard ratios for comparing the efficacy or safety of therapies in noninferiority studies. Annals of Internal Medicine 163 (2), 127-134.

Volinsky, C. T., Raftery, A. E., 2000. Bayesian information criterion for censored survival models. Biometrics 56 (1), 256-262.

Wei, L., 1992. The accelerated failure time model: a useful alternative to the Cox regression model in survival analysis. Statistics in medicine 11 (14-15), 1871-1879.

Yuan, M., Lin, Y., 2006. Model selection and estimation in regression with grouped variables. Journal of the Royal Statistical Society: Series B (Statistical Methodology) 68 (1), 49-67.

Zhang, Z., Sinha, S., Maiti, T., Shipp, E., 2016. Bayesian variable selection in the aft model with an application to the seer breast cancer data. Statistical Methods in Medical Research, To appear.

Zou, H., Hastie, T., 2005. Regularization and variable selection via the elastic net. Journal of the Royal Statistical Society: Series B (Statistical Methodology) 67 (2), 301-320. 